\title{
Numerically restricted food intake in the rat in a free-feeding situation
}

\author{
HANK DAVIS and SHEREE ANNE BRADFORD \\ University of Guelph, Guelph, Ontario, Canada
}

\begin{abstract}
Rats were trained to leave an array of food after they had consumed a fixed number (three, four, or five) of food items. This discrimination remained in effect despite a shift from 45-mg Noyes pellets to larger and irregularly sized sunflower seeds. The present demonstration replicates work first reported 50 years ago by Otto Koehler and his associates (e.g., Marold, 1939; see also Koehler, 1950), who used avian subjects, and, like those studies, it involved both positive and negative social reinforcement. The present procedure provided a rare instance in which numerical control was exerted over behavior that was primarily consummatory in nature. The results are viewed in terms of the wider literature of numerical competence in animals and are discussed in terms of a numerical process known as protocounting (Davis \& Pérusse, 1988b).
\end{abstract}

Under natural conditions, an animal tends to eat until it is sated, until it is forced by another animal to leave the food supply, or until the food source has been depleted.

In the present report, we describe a situation in which an animal has been trained to leave and return to a food supply, so that the size of each meal is regulated by the number of items the animal has consumed. The prototype for this numerical discrimination comes from work performed by Otto Koehler and his associates in the 1930s (see Koehler, 1950; Marold, 1939). In these studies, budgerigars were trained to eat a fixed number of grains (ranging between two and six) from randomly sized arrays of grain that exceeded each bird's "target number."

Koehler's studies have been essential to the development of the "animal counting" literature (e.g., Chauvin, 1988; Davis \& Memmott, 1982; Honigmann, 1942; Salman, 1943), despite the fact that the procedural details are not readily available in English. Moreover, the methodology used by Koehler and his associates has occasionally been criticized for possible confounding by nonnumerical cues (see Wesley, 1961). Our goal in the present paper was to provide a well-controlled replication of Marold (1939) and Koehler's (1950) demonstration, using a mammalian species (Rattus norvegicus) to show that behavior that is essentially consummatory can be regulated by numerical cues in the manner typically associated with instrumental responding.

These data were presented in various forms at meetings of the Animal Behavior Society (1987) and the Psychonomic Society (1986). The research was supported in part by Grant A0673 from the Natural Sciences and Engineering Research Council of Canada to $H$. Davis. We are grateful to Rachelle Perusse and Associate Editor Marcia Spetch for their critical comments. Correspondence should be addressed to Hank Davis, Department of Psychology, University of Guelph, Guelph, Ontario NIG 2W1, Canada.

\section{METHOD}

\section{Subjects}

Ten female Long-Evans rats served as subjects. All animals were approximately 120 days old at the start of training. The subjects were housed individually with water available in their home cages. During initial training, all animals were maintained at minimal food deprivation, ranging between $90 \%$ and $95 \%$ of their free-feeding weights. Subsequently, free food was available in the home cage.

\section{Apparatus}

The apparatus consisted of a start area (the $40 \times 40 \mathrm{~cm}$ seat of an upholstered chair) and a 20 -cm-wide wooden plank, which extended $6 \mathrm{~m}$ from the edge of the start area.

\section{Procedure}

All animals were run for two sessions a day during all of the following phases.

Habituation. Each animal was handled for 10-20 min a day by the experimenter for $\mathbf{4}$ days prior to habituation. At the start of each habituation session, each animal was placed on the plank and allowed to explore freely and discover and consume several 45-mg Noyes pellets. An average of five habituation sessions, approximately $20 \mathrm{~min}$ each, were held. The experimenter crouched by the side of the plank and talked during all sessions, in order to familiarize the animals with taking food from the experimenter's hand, as well as with the sound of the experimenter's voice.

During the final sessions, the edge of the plank was connected to the underside of the chair so that the animal could gain access to the plank by jumping down approximately $7 \mathrm{~cm}$. The animals were shaped to explore the plank and were manually rewarded with food for returning to the start area after consuming food on the plank.

Training Phase 1. Each animal was randomly assigned to a target number condition. Four animals were designated as "3-eaters," 3 animals as "4-eaters," and 3 animals as "5-eaters." Each animal was placed on the seat of the chair (start area) at the beginning of a trial and allowed to explore the plank. An array of 45-mg Noyes pellets was located between 0.5 and $1.0 \mathrm{~m}$ from the edge of the start area. Initially these arrays consisted only of the target number. Eventually, arrays were increased to between one and five pellets more than the animal's target number (e.g., for a " 3 " rat, there were between four and eight pellets available on the plank) 
The target amount of food was initially separated from the remaining pellets by approximately $10 \mathrm{~cm}$. This separation was progressively eliminated over five sessions.

A "correct" trial consisted of the animal consuming its target number of pellets, leaving the remaining food uneaten, and returning to the start area. Correct performance was verbally praised by the experimenter (situated approximately $0.5 \mathrm{~m}$ away, beside the plank) as soon as the animal had turned from the pellets and was returning to the start area. These animals were further reinforced with praise and petting, as well as one additional Noyes pellet, after they had returned to the start area.

Any attempt to eat more than the target number of pellets (operationally defined in terms of oral contact) was immediately punished by a verbal response ("No!") and a handclap from the experimenter. Such a consequence typically caused the animal to stop eating and run from the plank back to the start area on the chair. The intensity of punishment was adjusted both within and between animals, to find a level that was sufficiently aversive to suppress incorrect performance (i.e., eating more than the target number), while not inhibiting exploratory or consummatory behavior. When animals consumed fewer than the target number of pellets, they received neither punishment nor further reward (praise and additional food were withheld on return to the start area).

The location of the experimenter was gradually changed over sessions so that she stood behind the start area; thus, animals moved in a direction away from the experimenter as they traversed the plank, and ran back toward her when they returned to the start area after completing a trial. Phase 1 of the procedure was maintained for approximately 10 trials per day ( 5 per session) over a 10-day period.

Training Phase 2. The number of pellets available on each trial was increased to 15-20. The pellets were presented in arrays that varied randomly in size and pattern across sessions. Approximately 15-20 sessions (five trials per session) were run in Training Phase 2. By the end of Phase 2, each animal reliably initiated trials by jumping from the start area to the plank, and also demonstrated completion of the trial by climbing back onto the start area after consum- ing no more than the target number of pellets, or being punished for exceeding this number.

Testing Phase 1. Data collection was begun in Testing Phase 1. Each session consisted of four trials and took approximately $5 \mathrm{~min}$ to complete. Correct responses and errors were recorded and continued to be rewarded and/or punished as before, with the exception that verbal praise was no longer delivered during a trial as it had been during the training phases. Following each trial, regardless of its outcome, the animal was required to return to the start area in order to "reset the counter to zero" and signal the end of a trial.

Pellet arrays were located between 0.5 and $1.0 \mathrm{~m}$ from the start area and consisted of 15-20 pellets on the initial trial of each session. Pellets were located approximately $3 \mathrm{~cm}$ apart on the average, with interpellet space ranging between $8 \mathrm{~mm}$ and $5 \mathrm{~cm}$. Because the animal progressively reduced the number of pellets remaining on each trial, it was occasionally necessary to rebait the plank between the trials in a session. On occasion, the final trial consisted of an array comprising the target number +1 pellet, thus requiring the animal to exit the test area with a single food item remaining. The Phase 1 procedure was continued for approximately 200 sessions.

Testing Phase 2. The procedure remained essentially unchanged, except for the substitution of irregularly sized, shelled sunflower seeds for 45-mg Noyes pellets. A sample of 100 seeds ranged in weight from 23 to $97 \mathrm{mg}$, with an average weight of $69.3 \mathrm{mg}$ (approximately $50 \%$ greater than that of Noyes pellets). The subjects had no experience with sunflower seeds as a food item prior to exposure in Phase 2. Phase 2 was maintained for approximately 25 sessions (100 trials).

Testing Phase 3. All aspects of the procedure remained the same, except for removal of the experimenter from the testing room. During Phase 3, the experimenter was situated in an adjoining room, from which the animal's performance could be observed over a video monitor. During the initial two sessions, the experimenter reentered the test area to give performance feedback (praise/petting/ additional pellets) only after completion of a session. An attempt was made to gradate such reward, depending on the proportion of

Table 1

Percentage of Trials on Which Each Subject Consumed Fewer Than, Exactly, or More Than the Target Number of Food Items

\begin{tabular}{lcccc}
\hline Subject & $\begin{array}{c}\text { Final 25 Trials } \\
\text { Phase 1 }\end{array}$ & $\begin{array}{c}\text { Initial 25 Trials } \\
\text { Phase 2 }\end{array}$ & $\begin{array}{c}\text { Final 25 Trials } \\
\text { Phase 2 }\end{array}$ & $\begin{array}{c}\text { Final 25 Trials } \\
\text { Phase 3 }\end{array}$ \\
\hline & & 3-Eaters & & \\
S22 & $12 / 72 / 16$ & $24 / 60 / 16$ & $8 / 76 / 16$ & $8 / 68 / 24$ \\
S23 & $8 / 64 / 28$ & $24 / 48 / 28$ & $8 / 72 / 20$ & $12 / 68 / 20$ \\
S24 & $12 / 60 / 28$ & $28 / 40 / 32$ & $8 / 64 / 28$ & $8 / 64 / 28$ \\
S25 & $8 / 68 / 24$ & $24 / 60 / 16$ & $8 / 72 / 20$ & $8 / 72 / 20$ \\
$M$ & $10 / 66 / 24$ & $25 / 52 / 23$ & $8 / 71 / 21$ & $9 / 68 / 23$ \\
& & $4-$ Eaters & & \\
S26 & $8 / 60 / 32$ & $28 / 44 / 28$ & $8 / 64 / 28$ & $12 / 60 / 28$ \\
S28 & $12 / 64 / 24$ & $24 / 44 / 32$ & $12 / 68 / 20$ & $8 / 64 / 28$ \\
S29 & $12 / 56 / 32$ & $28 / 40 / 32$ & $12 / 60 / 28$ & $12 / 64 / 24$ \\
$M$ & $11 / 60 / 29$ & $27 / 43 / 31$ & $11 / 64 / 25$ & $11 / 63 / 27$ \\
& & $5-$ Eaters & & \\
S27 & $12 / 60 / 28$ & $28 / 44 / 28$ & $12 / 60 / 28$ & $12 / 64 / 24$ \\
S30 & $20 / 52 / 28$ & $32 / 40 / 28$ & $20 / 56 / 24$ & $16 / 56 / 28$ \\
S31 & $12 / 64 / 24$ & $24 / 48 / 28$ & $16 / 64 / 20$ & $16 / 68 / 16$ \\
$M$ & $15 / 59 / 27$ & $28 / 44 / 28$ & $16 / 60 / 24$ & $15 / 63 / 23$ \\
\hline
\end{tabular}

Note-Values in each column represent fewer than/target number/more than. The group means are rounded to nearest whole integer. 
correct trials in the session, with particular attention to performance on the final trial. This procedure was altered for the remaining sessions in Phase 3. While located in an adjoining room with the door slightly ajar, the experimenter again punished incorrect performance on a trial-by-trial basis by shouting and hand clapping. This feedback was audible despite the physical distance between the experimenter and the subject. Phase 3 conditions were maintained for a total of 10 sessions (40 trials).

Randomly selected sessions from all subjects during Testing Phases 1-3 were videotaped and scored by an independent observer, in order to confirm the performance data recorded by the experimenter. Interobserver reliability equaled or exceeded 0.9 over all cases.

\section{RESULTS}

For the sake of analysis, performance was grouped into three categories: trials on which the subject consumed the target number (correct trials); trials on which the subject

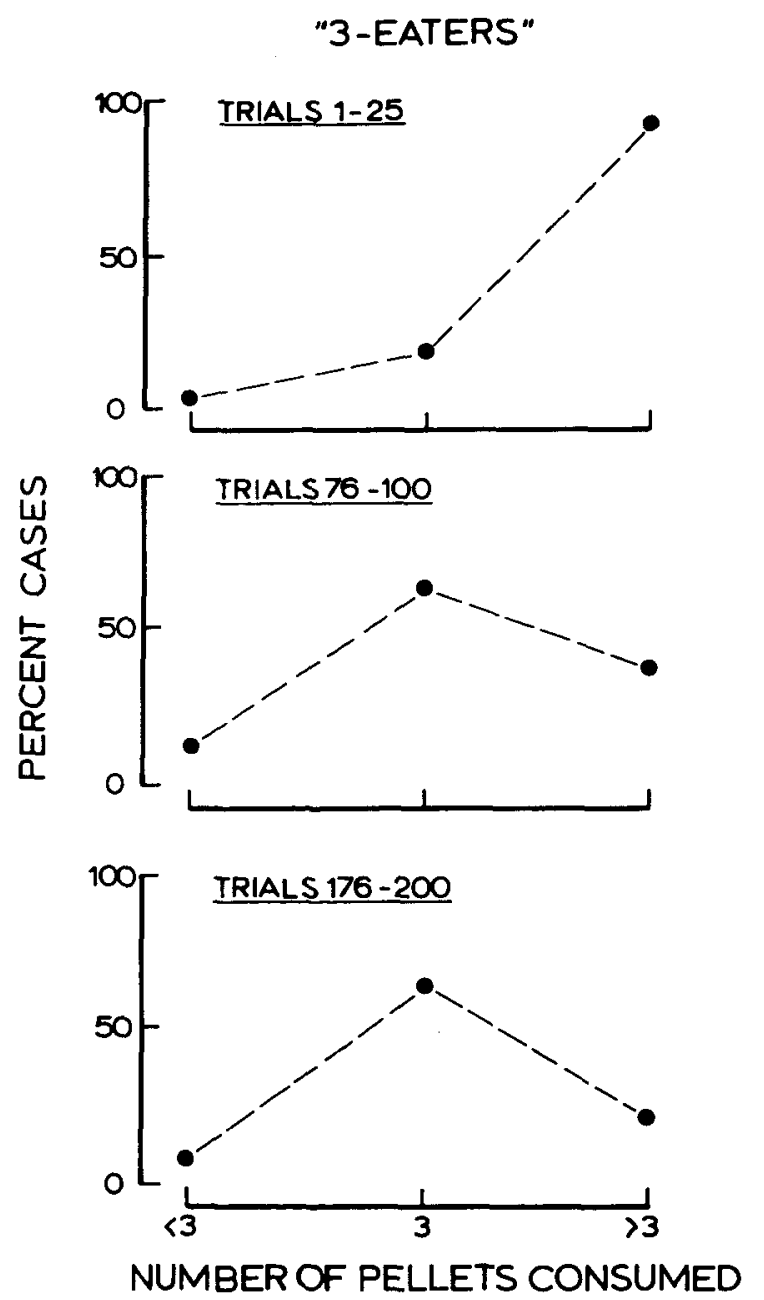

Figure 1. Mean percentage of trials on which subjects in the 3eater condition ate fewer than, exactly, or more than the target number of pellets during various stages of training in Phase 1 .
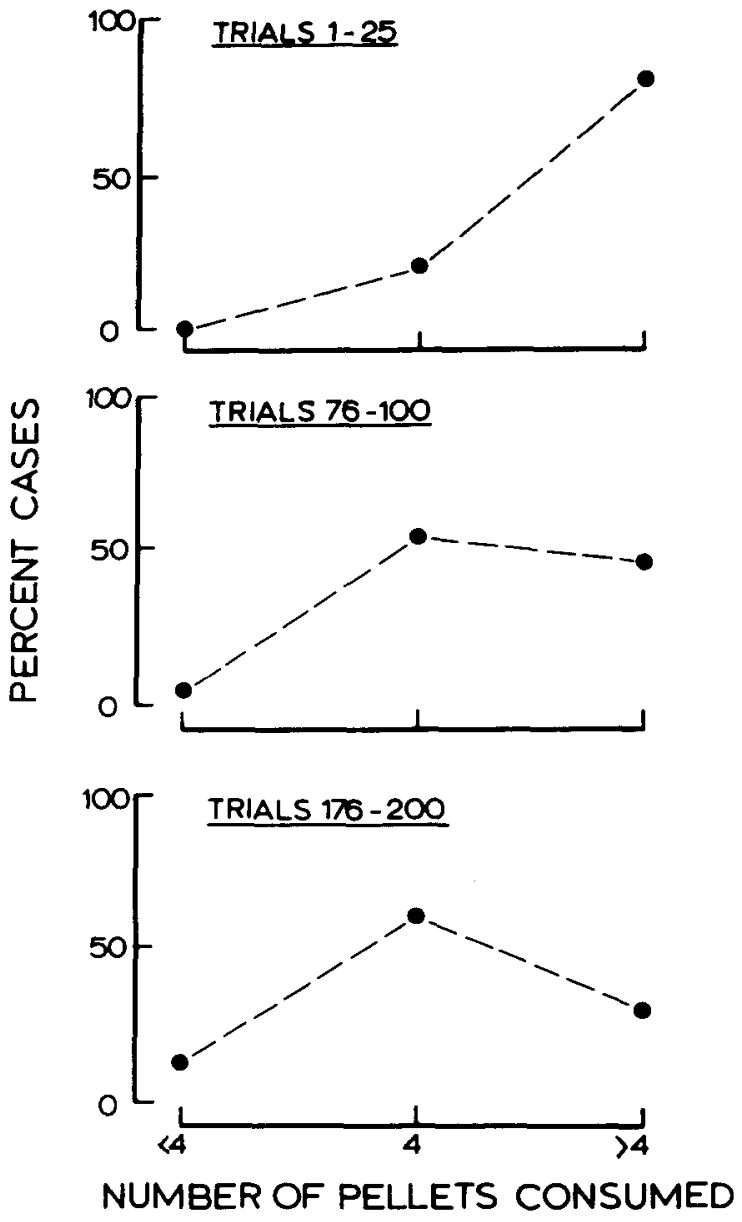

Figure 2. Mean percentage of trials on which subjects in the 4 eater condition ate fewer than, exactly, or more than the target number of pellets during various stages of training in Phase 1 .

ate fewer than the target number of pellets; and trials on which the subject ate more than the target number.

\section{Phase 1}

The final Phase 1 performance of all subjects is summarized in Table 1 . In every case, the modal number of pellets eaten per trial corresponded to the subject's target number; for example, on the trials, subjects trained to eat only three pellets ate three more often than they did fewer or more than three. Use of the binomial probability table to evaluate these results reveals that the tabled performance of all subjects in Phase 1 was significant at the .05 level. ${ }^{1} \mathrm{~A}$ Kruskal-Wallis one-way analysis of variance (ANOVA) performed on data from the final trial block in Phase 1 revealed no difference in the accuracy of performance between the three target-number conditions $(H=2.89, p>.25)$.

All subjects showed a gradual change in the distribution of outcomes over the course of training. The progressive shift in modal responding to the target number is il- 


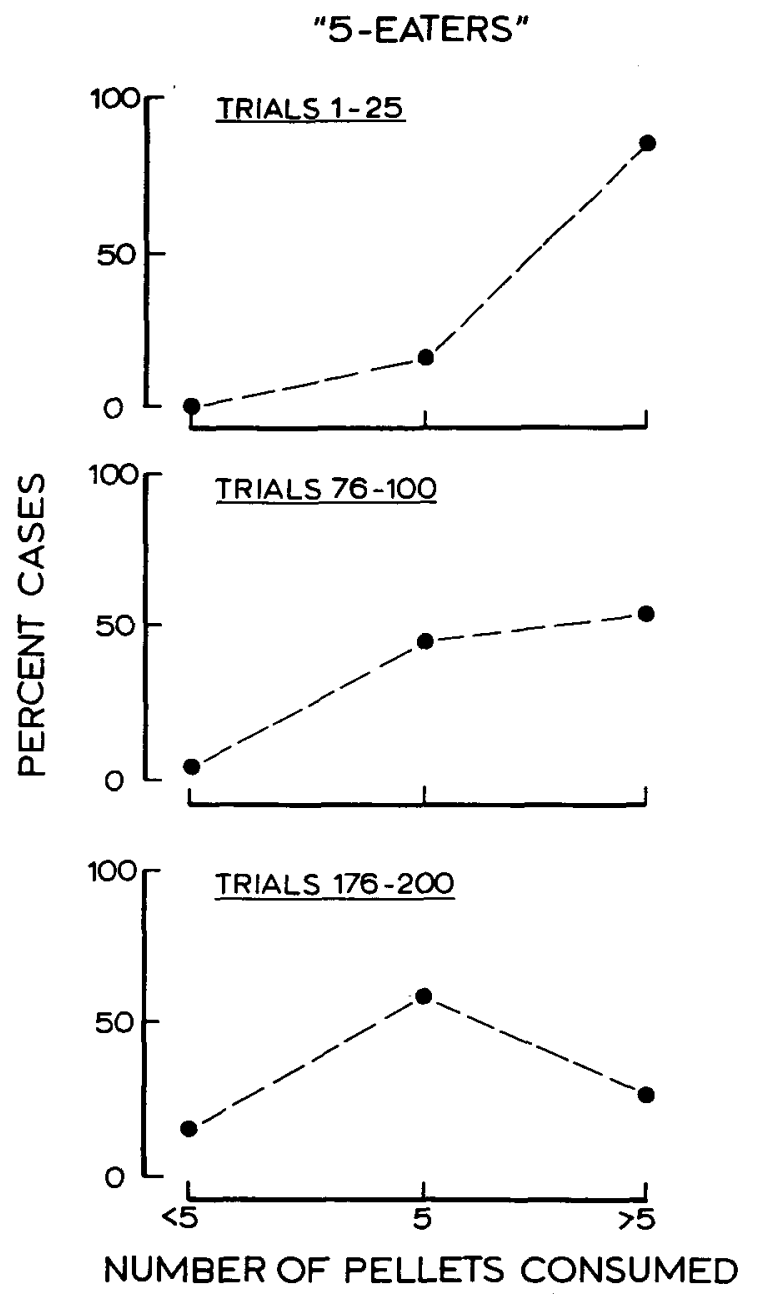

Figure 3. Mean percentage of trials on which subjects in the 5eater condition ate fewer than, exactly, or more than the target number of pellets during various stages of training in Phase 1 .

lustrated by mean performance curves obtained under each of the three numerical requirements. An examination of these data in Figures 1-3 confirms that numerical control was sharpened with increased training. All subjects began by eating virtually all of the available food; there was no tendency to restrict the size of the meal. Increased training caused the subjects to attend to the number of items they consumed and thereby regulate food consumption. As training progressed, there were occasions on which the subjects underestimated the numerical requirement; that is, the subjects ate fewer than the required numbers of items. Although such cases are noteworthy, they never constituted the majority of incorrect trials. By far the most frequent errors resulted from overeating-that is, from consuming more than the target number of pellets and receiving punishment.

To emphasize the role of numerical cues, we were particularly careful about the pattern in which we presented the food items. Although we attempted to guard against employing symmetrical or consistent arrays, our concern appeared to be unnecessary. Figure 4 illustrates 1 rat's performance on probe trials involving symmetrical pattern cues that might have facilitated correct performance. Note that in these cases, the animal's selection of pellets did not conform to a simple figural pattern. Moreover, subsequent trials that provided the animal with an opportunity to consume food in the same figural sequence rarely if ever yielded such consistency. In short, pattern cues do not appear to have played an essential role in the regulation of food consumption.

There was considerable variability in trial duration, both within and between subjects. Overall trial duration included three components: time between leaving the start area and eating the first food item; time between eating the first and last food items (eating time); and time between eating the final item and returning to the start area. We were concerned only with eating time, not with travel to and from food. Two randomly chosen subjects under each of the three numerical requirements were examined for the relationship between eating time and outcome. For this analysis, 30 consecutive video-recorded trials from each subject were sorted into fewer than target, more than target, and correct outcomes. A Kruskal-Wallis ANOVA was performed to determine whether these three outcomes differed with regard to their underlying eating times. No such relationship was found; thus, for example, rats were not more likely to make fewer-than-target errors when they ate slowly, or more-than-target errors when they ate rapidly $(H=0.308, p>.50$, for the 3 -eater condition; $H=0.228, p>.50$, for the 4-eater condition; $H=$ $0.396, p>.50$ for the 5 -eater condition).

The numerical requirement was not itself a major determinant of the speed of performance; there were many

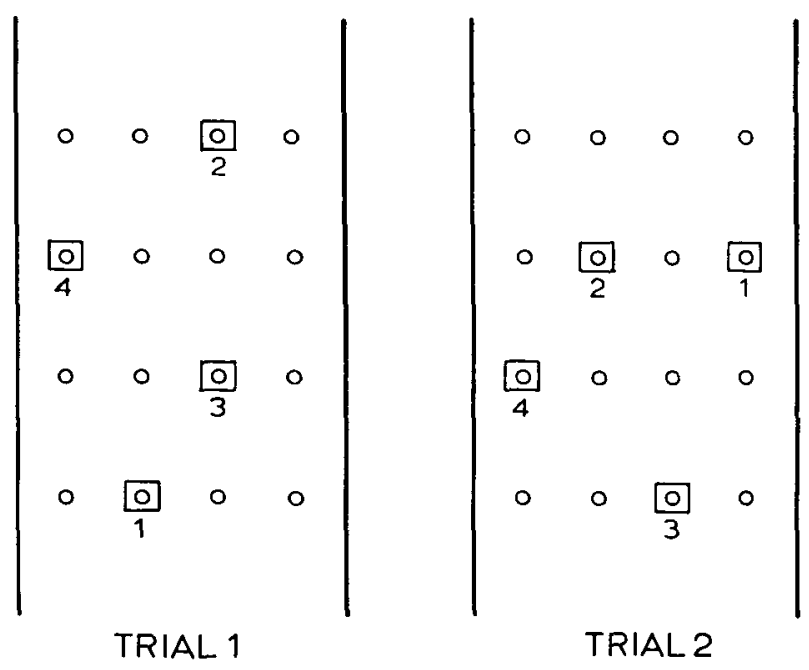

Figure 4. Sequence of pellets consumed by a 4-ater on two consecutive probe trials with replacement of items between trials. 


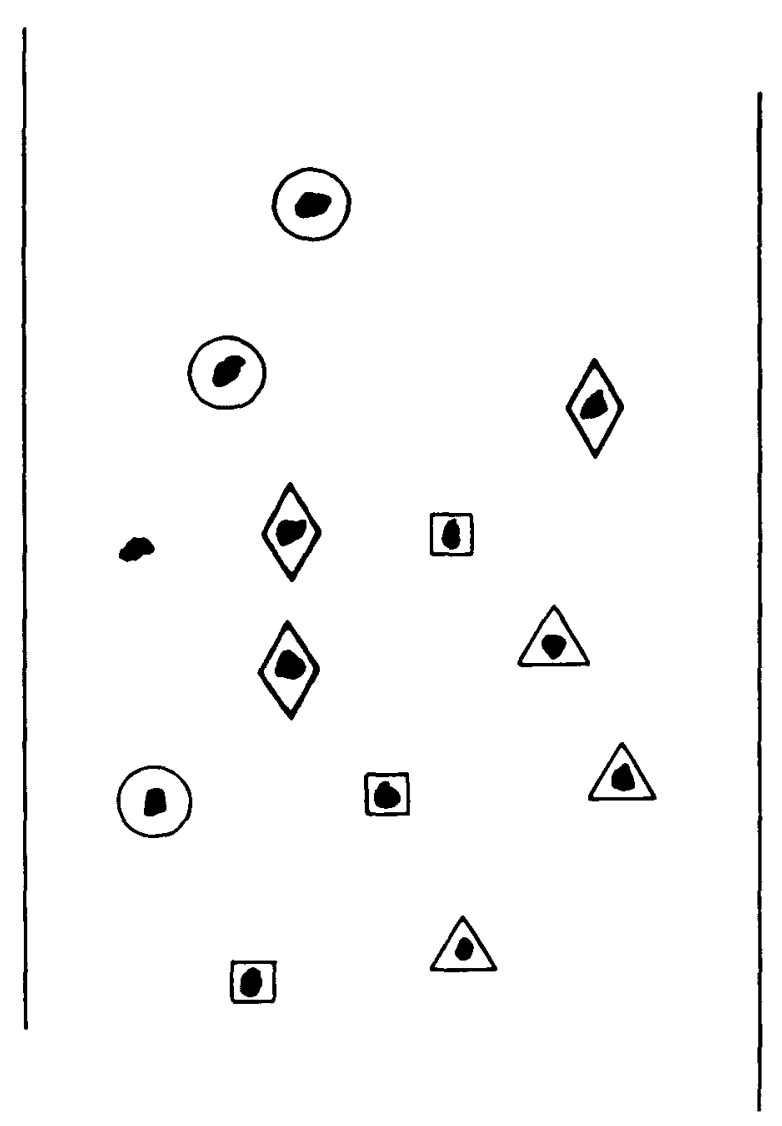

Figure 5. Correct performance by a 3-eater on four consecutive trials with no replacement of food items between trials. Trial 1 selections are indicated by $\square$; Trial 2 by $\Delta$; Trial 3 by 0 ; Trial 4 by $O$.

instances, for example, in which 3-eater trials required more time to complete than the 5-eater trials did. A comparison of correct eating times for randomly chosen subjects under each numerical condition revealed no significant group difference (Kruskal-Wallis $H=0.534$, $p>.50$ ). In addition to overall trial durations, interpellet intervals within trials did not support the notion that performance was controlled by a "rhythmic" pattern.

At each of the three numerical requirements, there were occasions on which a correct response required the animal to exit the plank, leaving behind a single piece of food uneaten (see Figure 5). Such trials, though they were given no additional weight in the analysis, may be more intuitively persuasive in underscoring the strength of this numerical discrimination.

\section{Phase 2}

The data from the first and final trial blocks in Phase 2 are presented in Table 1. The changeover from $45-\mathrm{mg}$ Noyes pellets to larger and irregularly sized sunflower seeds had a transient disruptive effect on performance. The fact that successful performance was reestablished by the third session suggests that discrimination was based on numerical cues, rather than volumetric or size-related information. Indeed, during the initial changeover in Phase 2, animals frequently appeared "disoriented" and occasionally failed to consume any food, or they took just a single piece of the novel food item before exiting. Once discriminative control was reasserted, however, performance was maintained unchanged from the levels reported for Phase 1.

\section{Phase 3}

Phase 3 conditions were tested to confirm that performance did not depend on cuing by the experimenter. The data from Phase 3 are presented in Table 1. In general, significant $(p<.01)$ performance was maintained despite the experimenter's absence from the test enclosure. However, there were some notable exceptions.

During the first two sessions in Phase 3, a number of cues suggested that the experimenter was no longer in the room. In addition to direct sensory evidence of her absence, two procedural differences were also detectable: (1) Performance was no longer rewarded following each trial-that is, when subjects returned to the start area between trials in order to "reset the counter," there was no contact with the experimenter; and (2) immediate punishment (shouting, handclapping) no longer occurred when an error was made. Following the second session in Phase 3, a procedural modification permitted the experimenter to deliver punishment for errors. However, the animals' performance in the initial two sessions is worth describing in detail.

The absence of reward following correct trials was not particularly disruptive; subjects proceeded to the next trial after only a brief delay. However, the absence of punishment following an error had a marked effect on performance. Consider the following record of $\mathbf{2 2 3}$, a 3-eater, whose performance is representative of that observed during the initial session in Phase 3.

Trial 1. The subject climbed to the plank and faced a random array of 15 pellets. After consuming 3, the subject returned to the start area.

Trial 2. After several seconds, the subject again climbed to the plank and consumed three pellets. After returning to the start area, the subject stood on its hind legs and repeatedly sniffed the air.

Trial 3. The subject climbed to the plank and, before eating any pellets, again stood on its hind legs and sniffed. The subject then consumed three pellets but did not return to the start area. The subject again stood and sniffed the air, and then slowly ate a fourth pellet. The subject then returned hurriedly to the start area.

Trial 4. The subject slowly walked along the plank and ate three pellets. The subject then stood on her hind legs, sniffed the air, and consumed a fourth pellet. After a brief pause, the subject rapidly consumed the remaining pellets on the plank and ran back to the start area. 
This performance is generally consistent with the accuracy rates observed for all subjects in the experiment. With two exceptions (S26 and S30), all subjects correctly consumed the target number of pellets on Trial 1. On Trial 2, all but 3 subjects (S24, S27, and S29) consumed their respective target numbers. On Trial 3, without performance feedback, half the subjects consumed more than the target number of pellets. By Trial 4 , only 1 subject restricted food intake to the target number.

On the following day (Session 2), Subject 23, as well as every other animal, had exceeded target and consumed most of the pellets on the plank by the end of Trial 3 . Performance appeared to have reverted to its original, pretraining level; but was the failure discriminative (i.e., numerical) or motivational? In the subsequent test session (Phase 3, Session 3), the experimenter again monitored trial-by-trial performance through a video camera and, although absent from the room, provided punishment (shouting, handclapping) of errors. Within two sessions, performance was reinstated to the significant levels recorded during the final Phase 2 sessions (see Table 1).

\section{DISCUSSION}

We have demonstrated that rats, originally inclined to eat all the food available in a test situation, can learn to restrict the size of each meal until each animal's modal response equals its target number.

The present procedure allowed us to control for a number of potentially confounding factors identified by Wesley (1961) in his criticism of studies described by Koehler (1950). For example, the fact that numerical control was retained despite the shift from regularly sized Noyes pellets to irregular and somewhat larger sunflower seeds underscores the numerical rather than size or volumetric basis of performance. It is also important that the results of Phase 3 allow us to preclude the role of a "Clever Hans" effect-that is, of discriminative cuing by the experimenter. In addition, the irregular temporal properties of performance make it unlikely that success was based on "rhythm," a confound present in much early "animal counting"' work (see Davis \& Pérusse, 1988b; Salman, 1943; Wesley, 1961).

This demonstration of numerical competence in rats provides a detailed confirmation of the success of a technique first established with avian subjects over 50 years ago. The procedural details and results of this original report have only been available in summary form to an English speaking audience (e.g., Koehler, 1950; Salman, 1943).

The present results are important for two additional reasons.

1. They extend the range of behaviors over which numerical cues may exert control. Most demonstrations in the numerical competence literature with animals involve modification of arbitrary instrumental responses rather than consummatory acts (see, e.g., Boysen $\&$ Berntson, 1989; Davis, 1984; Davis \& Albert, 1986; Fernandes \& Church, 1982). In drawing this distinction, we concede that the absolute classification of instrumental as opposed to consummatory behavior is not without problems, both within appetitive conditioning (e.g., Jenkins \& Moore, 1973) and in aversive control (e.g., Davis \& Shattuck, 1980). In the present situation, for example, the head movements used to procure food may have involved an instrumental component. However, we argue that in contrast to virtually all modern demonstrations of numerical competence, the behavior that was targeted for discriminative control in the present experiment may be viewed as essentially, if not absolutely, consummatory in nature (see also DeCosta \& Ayres, 1971).

2. The present results involve the use of rat subjects rather than the avians employed in the original work (Marold, 1939). Much historical work on "counting animals" was performed with birds-although with few exceptions (e.g., Pepperberg, 1987), rats have begun to appear as subjects more frequently in the modern literature (e.g., Capaldi \& Miller, 1988; Davis \& Bradford, 1986; Davis, MacKenzie, \& Morrison, 1989). Because of obvious differences in the foraging behavior of these two animal groups, it was not a forgone conclusion that the positive results previously found with birds would be replicated with rats.

The performance of our subjects was inferior to that typically associated with simple discrimination learning in rats (cf. Munn, 1950); however, our results correspond to the numerical performance summarized by Koehler (1950). In terms of absolute success rates, our subjects ranged from a mean of $58.7 \%$ correct for the 5-eater condition to $66 \%$ for the 3-eater condition. Insofar as comparable data are available, we can note that Marold (1939) reported success rates in the $55 \%-60 \%$ range after 700 trials.

A notable species difference between our rats and earlier avian subjects exists in the salience of visual cues. For example, Marold (1939) reported that her subjects' performance was strongly influenced by the patterns in which food items were displayed. For example, performance of a subject trained by Marold to eat two items from a row containing between three and seven items declined from $87 \%$ to $44 \%$ correct when interitem spacing was reduced. In contrast, rat subjects in the present experiment were notably unaffected by pattern cues. Their performance was not disrupted by trial-to-trial variation in the spacing of food arrays, nor did the subjects show repetition in the pattern with which they consumed the items. The subjects typically failed to employ consistent or obvious strategies even when exposed to simple rows of food.

Like Koehler's work, our research involved a somewhat unconventional approach to both deprivation and reward. Regarding deprivation, Marold (1939) noted that training was more successful in what she termed "pre- 
fed" birds. Performance of her " very hungry" subjects frequently deteriorated even after extensive training. Our experience with rats was similar; $90 \%-95 \%$ deprivation levels were sufficient to train performance, which was then maintained without further deprivation. Infrequent occasions on which deprivation exceeded $10 \%$ ad-lib weight typically resulted not in discrete "errors" per se, but in total reversion to pretrained patterns of behavior.

Regarding the use of reward, our experiment involved a combination of supplemental feeding as well as praise and petting. Davis and Pérusse (1988a) have recently reported that human-based social interaction may be a powerful adjunct to conventional food reward in the training of rat subjects. However, our training method also involved the use of punishment (shouting, handclapping) to suppress incorrect responses. Indeed, the results of Phase 3 suggest that successful numerical discrimination depended on the continued presence of punishment for errors (see Davis, 1989, for related discussion). It is important to stress, however, that subjects did not simply eat until they were punished. Such trials counted as errors and would have resulted in a zero success rate. Rather, successful performance appeared to depend on maintenance of the punishment contingency itself, rather than on continuous delivery of punishment.

Like our own work, numerical training procedures reported by Koehler (1950), Loegler (1959), Marold (1939), and Braun (1952) also involved the use of unorthodox punishment techniques, often spontaneously dispensed by the experimenter. For example, Koehler (1950) reported "shooing away" birds after incorrect responses. Marold (1939) used blasts of air, in addition to spraying water into the faces of her birds when they were incorrect. Braun (1952) punished subjects by "making loud noises," throwing a wet sponge, or pulling the subjects' tail feathers. Although unconventional by contemporary standards, and admittedly difficult to quantify, these techniques are certainly no less natural than electric shock and were reasonably effective in establishing behavioral control.

As it did for food deprivation, our research suggests that performance was optimized when the intensity of punishment was minimal. Experience with pilot subjects revealed that without careful monitoring, the level of "social punishment" often appeared excessive, resulting in nearly complete suppression of both exploration and eating.

Although our data demonstrate another instance of numerical competence in the rat, they share an unfortunate characteristic with much of the numerical work with nonhuman subjects to date: they do not allow us to isolate the mechanism by which the numerical discrimination was made. In terms of the analysis proposed by Davis and Pérusse (1988b), the present procedure does preclude alternative numerical processes such as relative numerousness judgment, subitizing, rhythm, or estimation. However, the basic question frequently raised by numerical competence studies of animals remains: Are they counting? If viewed in terms of the criteria we have extrapolated from work with human subjects (Davis \& Memmott, 1982; Davis \& Perusse, 1988b), the present report does contain some suggestive evidence. For example, we describe the gradual and progressive consumption of individual items from an array, a process that may be seen as analogous to "partitioning and tagging," which is an important feature of counting (see Gelman \& Gallistel, 1978).

Although the subjects in the present experiment behaved as if they were using a cardinal chain in order to enumerate (and consume) items, there is no independent evidence to identify such serial tags or confirm their application in 1:1 correspondence to items in the array. The lack of this important prerequisite to "true" counting in most numerical competence work with animals underscores Koehler's (1950) observation that animals behave as if they are "thinking unnamed numbers." In short, the present results are best described in terms of what Davis and Pérusse (1988b) have termed protocounting, a category reserved for findings that strongly suggest, but do not yet provide, definitive evidence of "true counting."

\section{REFERENCES}

Boysen, S. T., Berntson, G. G. (1989). Numerical competence in a chimpanzee (Pan troglodytes). Joumal of Comparative Psychology, $103,23-31$

BRAUN, H. (1952). Über das Unterscheidungsvermögen unbenannter Anzahlen bei Papageien. Zeitung fur Tierpsychologie, 9, 40-91.

CAPAldi, E. J., Miller, D. J. (1988). Counting in rats: Its functional significance and the independent cognitive processes that constitute it. Journal of Experimental Psychology: Animal Behavior Processes, 14, 3-17.

Chauvin, B. (1988). Human infants are perhaps not so gifted after all. Behavioral \& Brain Sciences, 11, 583.

DAvis, H. (1984). Discrimination of the number three by a raccoon (Procyon lotor). Animal Learning \& Behavior, 12, 409-413.

DAvis, H. (1989). Theoretical note on the moral development of rats (Rattus norvegicus). Joumal of Comparative Psychology, 103, 88-90.

DAvis, H., ALBERT, M. (1986). Numerical discrimination by rats using sequential auditory stimuli. Animal Learning \& Behavior, 14, 57-59.

Davis, H., Bradford, S. A. (1986). Counting behavior by rats in a simulated natural environment. Ethology, 73, 265-280.

Davis, H., Mackenzie, K., \& Morruson, S. (1989). Numerical discrimination using body and vibrissal touch in the rat. Journal of Comparative Psychology, 103, 45-53.

Davis, H., * MEMmoTt, J. (1982). Counting behavior in animals: A critical evaluation. Psychological Bulletin, 92, 547-571.

DAvis, H., * Pérusse, R. (1988a). Human-based social interaction can reward a rat's behavior. Animal Learning \& Behavior, 16, 89-92.

Davis, H., \&ÉRusse, R. (1988b). Numerical competence in animals: Definitional issues, current evidence and a new research agenda. $B e$ havioral \& Brain Sciences, 11, 561-616.

Davis, H., SHatruCK, D. (1980). Transfer of conditioned suppression and conditioned acceleration from instrumental to consummatory baselines. Animal Learning \& Behavior, 8, 253-257.

DeCostA, M. J., \& AYres, J. J. B. (1971). Suppression of operant vs. consummatory behavior. Journal of the Experimental Analysis of Behavior, 16, 133-142. 
Fernandes, D. M., \& Church, R. M. (1982). Discrimination of the number of sequential events by rats. Animal Learning \& Behavior, 10, $171-176$.

Gelman, R., \& Gallistel, C. R. (1978). The child's understanding of number. Cambridge, MA: Harvard University Press.

HonigmanN, H. (1942). The number conception in animal psychology. Biological Review, 17, 315-337.

Jenkins, H. M., \& Moore, B. R. (1973). The form of the autoshaped response with food or water reinforcers. Joumal of the Experimental Analysis of Behavior, 20, 163-181.

KOEHLER, O. (1950). The ability of birds to "count." Bulletin of Animal Behaviour, 9, 41-45.

LOEGLER, P. (1959). Versuche zur Frage des Zähl-Vermögens an einem Graupapagei und Vergleichsversuche an Menschen. Zeitschrift für Tierpsychologie, 16, 179-217.

MAROLD, E. (1939). Versuche an Wellensittichen zur Frage des Zählvermögens. Zeitung für Tierpsychologie, 3, 170-223.

MUNN, N. L. (1950). Handbook of psychological research on the rat. New York: Houghton Mifflin.

Pepperberg, I. M. (1987). Evidence for conceptual quantitative abilities in the African grey parrot: Labelling of cardinal sets. Ethology, $75,37-61$
Salman, D. H. (1943). Note on the number conception in animal psychology. British Jourmal of Psychology, 33, 209-219.

Wesley, F. (1961). The number concept: A phylogenetic review. Psychological Bulletin, 58, 420-428.

\section{NOTE}

1. The binomial probability table actually offers a conservative test of performance, insofar as it assumes that the three outcomes (eating fewer than, more than, or exactly the target number of items) are equally probable. In reality, there are three, four, or five times as many opportunities to make a fewer than error under each of the three conditions, respectively, than there are opportunities for equal to or more than responses. Using this binomial analysis, trial blocks on which the target number is consumed on $48 \%$ of the cases or 12 or more times out of 25 has an associated $p$ value of less than .05 . Similarly, $56 \%$ or 14 correct out of 25 trials represent significant performance $(p<.01)$.

(Manuscript received February 18, 1989; revision accepted for publication April 11, 1991.) 